\title{
ACTEURS, RESSOURCES ET RÉSEAUX SOCIOLOGIQUES D'ÉCHANGES À PARTIR DE L'EXPÉRIENCE BELGE
}

\author{
Didier Vrancken
}

Presses de Sciences Po | Sociologies pratiques

\section{4/Supplément - HS 1}

pages 103 à 108

ISSN 1295-9278

Article disponible en ligne à l'adresse:

http://www.cairn.info/revue-sociologies-pratiques-2014-Supplément-page-103.htm

Pour citer cet article :

Vrancken Didier, “ Acteurs, ressources et réseaux sociologiques d'échanges à partir de l'expérience belge ", Sociologies pratiques, 2014/Supplément HS 1, p. 103-108. DOI : 10.3917/sopr.hs01.0103

Distribution électronique Cairn.info pour Presses de Sciences Po.

(C) Presses de Sciences Po. Tous droits réservés pour tous pays.

La reproduction ou représentation de cet article, notamment par photocopie, n'est autorisée que dans les limites des conditions générales d'utilisation du site ou, le cas échéant, des conditions générales de la licence souscrite par votre établissement. Toute autre reproduction ou représentation, en tout ou partie, sous quelque forme et de quelque manière que ce soit, est interdite sauf accord préalable et écrit de l'éditeur, en dehors des cas prévus par la législation en vigueur en France. II est précisé que son stockage dans une base de données est également interdit. 


\section{Acteurs, ressources et réseaux sociologiques d'échanges à partir de l'expérience belge}

Didier VRancken ${ }^{1}$

\section{Un paysage à l'origine peu favorable aux collaborations entre institutions}

En Belgique, le contexte institutionnel n'a guère été favorable au rapprochement entre sociologues universitaires (Vrancken, 2012), car la recherche et l'enseignement de la sociologie sont essentiellement organisés au sein d'universités, aux pouvoirs organisateurs très différents: autorités catholiques et ecclésiastiques (Université catholique de Louvain, Facultés Notre-Dame de la paix de Namur, Facultés universitaires Saint-Louis à Bruxelles, Facultés universitaires catholiques de Mons), pouvoirs publics (Université de Liège, Université de Mons), libre pensée et laïcité (Université libre de Bruxelles).

De ce fait, jusqu'à ces dernières années, les sociologues collaboraient relativement peu entre réseaux universitaires aux pouvoirs organisateurs très disparates, voire clivés. Ils se connaissaient, entretenaient des liens affinitaires, se témoignaient un certain respect mutuel, mais sans que l'on puisse parler de véritable dynamique de collaboration en matière de recherche et d'enseignement, même s'il faut faire mention de contacts et d'échanges au sein de l'Association des Sociologues de Langue Française (AISLF) ou de l'Association des Sociologues Belges de Langue Française (ASBLF) aujourd'hui dissoute.

Actuellement, la situation serait à ce niveau peut-être en pleine évolution. Avec la réforme dite "de Bologne ", des écoles doctorales thématiques interuniversitaires ont vu le jour en Communauté française de Belgique. Elles favorisent désormais les rapprochements et les collaborations en matière d'enseignement entre universités. Celles-ci, sous la houlette du Fonds national de la recherche scientifique (FNRS) sont désormais tenues de co-organiser les cycles de formation doctorale en sociologie. Par ailleurs, on observe qu'avec le développement de la recherche, la création de centres de recherche, la croissance du nombre d'étudiants en sciences humaines et sociales, des chercheurs, voire des professeurs passent de plus en plus souvent d'une université vers l'autre. Enfin, la récente création de l'Association belge francophone des sociologues et des anthropologues (ABFSA) a également contribué à démultiplier les rencontres et les échanges entre sociologues belges francophones. Signalons toutefois qu'aucune initiative collective n'a été prise en vue de raffermir les liens avec les sociologues néerlandophones du nord du pays.

1. Université de Liège. 
II apparaît ainsi que l'évolution du paysage universitaire sous l'effet de cette double contrainte (réforme du paysage universitaire de l'enseignement et réforme de la politique de la recherche) a sensiblement contribué à modifier l'état des relations entre les sociologues, sans perdre de vue qu'un décret - très controversé - de création de grandes académies universitaires est actuellement en préparation.

On peut donc tenter de dégager quelques traits des évolutions actuellement en cours.

\section{Des associations aux réseaux d'échanges}

Une hypothèse est que l'on assiste de plus en plus au développement d'arènes d'échanges entre sociologues belges. Mais ce mouvement se fait moins sur le modèle associatif - qui n'a jamais vraiment pris parmi les sociologues belges francophones que sur le modèle de réseaux d'échanges en matière d'enseignement et de recherche. Assez paradoxalement, on retiendra qu'en Belgique le modèle associatif était assez peu développé entre sociologues, dans la mesure où la société civile, de son côté, apparaissait très structurée autour des "piliers " ${ }^{2}$ socio-politiques et idéologiques (chrétien, socialiste-laïque et libéral). Dès lors la professionnalisation de la sociologie s'était largement développée de manière «invisible» (Charlier, 1995), et non problématisée à l'ombre de cette dynamique liée aux particularités de l'État social belge. Un État social, rappelons-le, largement adossé à des initiatives publiques mais également associatives et privées qui ont joué un rôle considérable dans l'intégration invisible des sociologues sur le marché du travail.

Aujourd'hui, de nombreux lieux, arènes de débat ou réseaux d'échanges où interviennent les sociologues belges sont identifiables.

a. Au niveau international, on peut signaler la présence de nombre d'associations telles que l'AISLF, l'Association internationale de sociologie (ISA), l'Association européenne de sociologie (ESA) où interviennent, souvent de manière indifférenciée, les sociologues belges. Toutes ces associations sont aujourd'hui gagnées par cette volonté de s'appuyer sur les nouveaux médias afin de permettre à la sociologie d'entrer en résonnance et en débat avec la Cité. On peut particulièrement penser au thème de la sociologie publique développé par l'ISA (public sociology) et à la volonté de l'AISLF de lui emboîter le pas.

b. On n'y a sans doute pas été assez attentif mais des associations internationales se sont développées pour faire de la sociologie française, anglo-saxonne ou allemande ou autre dans le respect de leurs sensibilités linguistiques et culturelles et éviter sans doute de voir ces spécificités sacrifier aux exigences de la culture dominante. On peut en trouver des exemples auprès d'associations telles que l'AISLF, I'Association turcophone de sociologie, le Conseil arabe des sciences sociales, etc.

c. On note bien évidemment aussi l'existence d'associations nationales en France, Suisse, Canada, Italie, Portugal, et, comme on l'a dit, en Belgique. Pour la plupart, ces

2. Ce modèle s'est forgé à partir de véritables conglomérats verticaux de la société civile regroupant partis politiques, syndicats, mutuelles, sociétés coopératives, réseaux d'enseignement, organisations d'éducation permanente, de santé, de loisirs, presse, etc. au sein de grandes familles au départ idéologiques (chrétienne, socialiste et libérale). 
associations entendent également s'ouvrir à des sociologues non nationaux. Les textes d'intention de leurs différentes manifestations scientifiques sont généralement adressés à un public débordant largement leurs frontières.

d. Les réseaux doctoraux sont présents dans la plupart des pays, voire organisés au sein d'associations internationales, comme par exemple le Réseau doctoral (Rédoc) au sein de l'AISLF. II faut remarquer qu'en Belgique francophone, le réseau doctoral a été un facteur important de rapprochement entre les universités. Ainsi le FNRS ne reconnaît-il les programmes doctoraux que lorsque les conférences et sessions proposées sont organisées par au moins deux universités francophones différentes!

e. Les associations professionnelles et réseaux de sociologues. II n'y a pas à proprement parler d'association professionnelle de sociologues en Belgique (même si l'ABFSA est très clairement ouverte aux sociologues extra-universitaires) mais on constate cependant l'existence de réseaux d'étudiants diplômés qui organisent des rencontres, des débats, des formations et échangent des informations quant au marché de l'emploi. L'Université de Liège a créé en 2007 une association très active d'anciens diplômés en sciences sociales.

\section{La « mondialisation » de la recherche et de l'enseignement : l'accentuation d'une évolution déjà ancienne}

L'ancrage de la sociologie belge francophone s'est rapidement opéré au sein de réseaux internationaux. Ceci s'explique non seulement par la faible collaboration entre universités mais aussi par la taille très modeste du pays. L'exiguité de son territoire a beaucoup contribué à pousser ses chercheurs à en franchir les frontières. Petite démocratie (small democracy) ayant joué un rôle clé en matière de mise sur pied d'organisations internationales, la Belgique a souvent vu nombre de ses chercheurs quitter le pays pour se former et développer leurs recherches ailleurs. En ce qui concerne la sociologie, on se souviendra que des Belges - et plus particulièrement Henri Janne - furent à l'origine de la création, en 1958, de l'AISLF. Sans y être bien évidemment tous présents, les sociologues belges y ont toujours assuré une présence active et ce, sans discontinuité, depuis cette période. Bien sûr, la France et les pays de la francophonie sont longtemps demeurés des pays d'échanges privilégiés. Toutefois, issus d'un cadre national où l'on ne compte pas moins de trois langues officielles (le néerlandais, le français, l'allemand), nombre de sociologues belges ont de longue date appris à évoluer dans le monde anglo-saxon, préférant nouer des contacts au-delà de l'espace francophone tant en matière de collaborations et de recherches internationales que de développement de réseaux scientifiques.

Au fond, ce mouvement s'est accentué au cours de ces dernières années. Car les jeunes générations de chercheurs, s'ils souhaitent faire carrière dans une université belge, savent très bien qu'ils n'obtiendront aucun poste s'ils ne publient pas dans des revues internationales (même avant la soutenance de leur thèse) et s'ils ne partent pas au moins six mois à l'étranger (condition sine qua non - et ce, de préférence dans un pays anglo-saxon). Les règles de recrutement pratiquées par les universités mais également la récente réforme du FNRS leur ont donné des signaux très clairs. Ces nouvelles 
orientations ont contribué à durcir les règles de promotion au sein de la carrière académique pour les professeurs en place.

\section{Les conséquences de cette mondialisation sur l'activité de recherche et d'enseignement}

Les conséquences de cette "mondialisation » sont multiples et vont d'ailleurs dans des sens très divers.

a. De jeunes chercheurs épousent ce mouvement, parviennent à obtenir des grades, à décrocher des bourses et des financements de bourses doctorales et/ou post-doctorales et apprennent à démultiplier les articles et les publications. En un sens, ce mouvement a contribué à l'émergence de véritables professionnels de la recherche internationale.

b. Toutefois, cette course produit aussi une précarisation de la recherche. Nombre de chercheurs "mangent de la vache enragée " depuis des années tant les postes demeurent rares. Leurs vies professionnelles, familiales et conjugales s'en ressentent fortement. Certains passent de CDD à CDD, sans pouvoir se stabiliser et mener à bien un projet de thèse. Des études commencent à s'intéresser à la précarisation du travail des chercheurs sur le marché du travail universitaire, dans un contexte de course à l'excellence et au ranking des universités. De nombreux jeunes chercheurs quittent la recherche, amers, déçus parce que leur patron de recherche ou l'université n'a « rien pu faire pour eux".

c. Ce mouvement ne doit pas être découplé du contexte social et économique européen. La Belgique est de plus en plus sollicitée par des demandes de post-doc en provenance des pays du Sud de l'Europe. Nombre de chercheurs de ces pays vont tenter leur chance dans d'autres pays européens. La crise produit des effets implacables sur la recherche internationale en sciences sociales.

d. Dans un pays où le financement des universités se fait au prorata du nombre d'étudiants, je ne puis témoigner que d'une chose: nous opérons sur un véritable marché concurrentiel où toute part de marché gagnée par une université se fait au détriment des autres. Dans ce contexte, on comprend combien la gouvernance de nos universités est désormais sensible à la question des classements des universités. En clair, cette approche du marché universitaire n'est pas uniquement déployée à l'échelle des États-nations, elle s'internationalise de plus en plus à travers des méthodes de comparaison et de benchmarking. La marchandisation pénètre peu à peu des espaces qui lui avaient longtemps échappé.

\section{Des recompositions professionnelles sur les flancs, mais une professionnalisation de la recherche internationale en sociologie}

À la lecture de ce qui précède, on retiendra qu'en Belgique la reconnaissance du métier de sociologue s'est essentiellement produite au sein des universités, peu en 
dehors. On peut postuler que l'évolution que nous connaissons actuellement avec le développement de nouveaux masters en sciences sociales ne va pas contribuer à une visibilité de ce métier aux multiples facettes (M. Legrand, J.-F. Guillaume et D. Vrancken). À ce propos, on peut citer trois masters qui sont actuellement mis en place : la gestion des ressources humaines, les sciences du travail, les études de la population et développement. Ces trois masters destinent généralement nos étudiants diplômés aux emplois dans les entreprises privées, publiques, les associations mais aussi dans les organes de coopération au développement, etc. II semble donc que la professionnalisation qui se produit opère plutôt sur les flancs (et non pas au cœur) de la sociologie, au sein de ces différents programmes d'études spécialisées où les étudiants mobilisent énormément de compétences et de savoirs théoriques et méthodologiques en provenance de la sociologie et des sciences sociales en général.

De leur côté, les études en sociologie et en anthropologie semblent plutôt destinées à produire cette nouvelle génération de « très bons professionnels de la recherche internationale ". II y a fort à parier que cette tendance à la spécialisation de la recherche va continuer à croître davantage, ce d'autant que les demandes de recherches adressées aux laboratoires universitaires n'ont cessé d'augmenter au cours de ces dernières années. Sans doute nos programmes d'études en sociologie et en anthropologie vont-ils progressivement évoluer vers la préparation d'étudiants-chercheurs évalués non plus sur base de connaissances théoriques mais à partir notamment de rédactions d'articles et de working papers. Remarquons toutefois que les programmes de sociologie et d'anthropologie préparant les étudiants à la recherche sont peu attractifs pour des étudiants désireux d'acquérir des formations universitaires professionnalisées.

\section{La sociologie et les nouvelles demandes extérieures de recherche et d'enseignement}

Le nombre de demandes de recherches, d'interventions ayant sensiblement augmenté depuis plus d'une vingtaine d'années, la sociologie se voit, dans ce contexte, de plus en plus sollicitée par des demandes de recherche fondamentale et appliquée.

Au niveau de l'enseignement, la réforme dite « de Bologne » et la mise sur pied de nouveaux masters en gestion des ressources humaines, en sciences du travail, en études de la population et développement n'ont pu être menées à bien qu'en y développant des stages, qui n'existaient guère dans nos universités. Ces trois masters semblent attirer des étudiants demandeurs d'études appliquées en sciences sociales. Ceux-ci sont souvent demandeurs d'exemples concrets, d'illustrations, de case studies, de stages, de nouvelles pédagogies, de relations avec le monde du travail et, de manière générale, avec le monde extérieur aux universités. Les relations avec ce monde extérieur et le marché du travail sont une des dimensions des évaluations internationales auxquelles nos facultés de sciences humaines et sociales sont désormais confrontées. La plupart des universités belges francophones ont, depuis 2005, dû se positionner, voire se restructurer en réfléchissant tant en terme d'enseignement que de recherche et en veillant désormais à leur articulation, là où il y a encore une dizaine d'années, cette articulation ne semblait guère pensée, à tout le moins ne paraissait pas poser problème. 
Toute l'importance accordée à l'« usager » des services publics - en l'occurrence l'étudiant - est aussi un formidable moyen de marchandisation et de libéralisation du monde universitaire, de pénétration, en somme, de milieux jusque-là relativement peu marchandisés.

\section{Références bibliographiques}

Charlier J.-E. et Scieur Ph., 1995, «En Belgique francophone », dans Legrand M., Guillaume J.-F. et Vrancken D. (eds), La sociologie et ses métiers, Paris, L'Harmattan, pp. 75-87.

Legrand M., Guillaume J.-F. et Vrancken D. (eds), 1995, La sociologie et ses métiers, Paris, L'Harmattan.

Vrancken D., 2012, "La sociologie en Belgique francophone au rythme des ordres négociés », Sociologies Pratiques, 24, pp. 137-148. 